\title{
An Algorithm for Determination of Rank and Degree of Contribution of sMRI Volumetric Features in Depression Detection
}

\author{
Kuryati Kipli and Abbas Z. Kouzani, Member, IEEE
}

\begin{abstract}
Brain volume changes at structural level appear to have utmost importance in depression biomarkers studies. However, these brain volumetric findings have very minimal utilization in depression detection studies at individual level. Thus, this paper presents an evaluation of volumetric features to identify the relevant/optimal features for the detection of depression. An algorithm is presented for determination of rank and degree of contribution (DoC) of structural magnetic resonance imaging (sMRI) volumetric features. The algorithm is based on the frequencies of each feature contribution toward the desired accuracy limit. Forty-four volumetric features from various brain regions were adopted for evaluation. From DoC analysis, the DoC of each volumetric feature for depression detection is calculated and the features that dominate the contribution are determined.
\end{abstract}

\section{INTRODUCTION}

Depression is the most common mental disorder worldwide, and currently the fourth largest contributor to the burden of disease as reported by the World Health Organization [1]. It is estimated that by 2020 , depression will remain a leading cause of disability, second only to cardiovascular disease [1]. Approximately 121 million people worldwide have been affected by depression [2]. Depression is associated with widely varying psychological and physiological features, and this heterogeneity is acknowledged within classification systems [3].

Diagnostic criteria for major depressive disorder (MDD) are currently based on clinical and psychometric assessment. Some widely used screening tests for the evaluation of depression include the Hamilton Rating Scale for Depression, Diagnostic Interview Schedule and Hospital Anxiety and Depression Scale.

In this paper, the brain structural MRI (sMRI) volumetric features are investigated to determine the most important features that contribute towards more accurate depression detection, whether a person is in depressed or non-depressed forms. The 3-D volumetric features are extracted from sMRI data provided by Neuropsychiatric Imaging Research Laboratory at Duke University named as Multisite Imaging Research In the Analysis of Depression (MIRIAD) [4]. To authors' knowledge, this is the first study

*Research supported by Deakin University HDR and MOHE SLAB scholarship.

K. Kipli is a Ph.D student at the School of Engineering, Deakin University, Waurn Ponds, Victoria, 3216, Australia. She is now on a study leave from Department of Electronic, Faculty of Engineering, Universiti Malaysia Sarawak, 94300 Kota Samarahan, Sarawak, Malaysia. (e-mail: kkipli@deakin.edu.au).

A. Z. Kouzani is with the School of Engineering, Deakin University, Waurn Ponds, Victoria, 3216, Australia (corresponding author: +61-352272818; fax: +61-3-52272167; e-mail: kouzani@ deakin.edu.au). that exploring the feature selection for depression classification from volume of multiple brain regions. The contributions of this paper include: (i) introduction of a new algorithm for determination of DoC of sMRI volumetric features, and (ii) evaluation and determination of the most discriminant sMRI volumetric features for single-subject classification of depression using the proposed algorithm.

This paper is organized as follows. Section II describes the related background. Section III presents the proposed algorithm. Section IV gives the experimental procedures. Section V presents the experimental results. Section VI provides discussion of the results. Finally, conclusions are given in Section VII.

\section{BACKGROUND}

Feature selection identifies the most useful features, and reduces the dimensionality whilst the most significant aspects of the data are represented by the selected features [5]. Feature selection can be summarized into three categories: filter, wrapper, and hybrid methods. The limitations of ranking and subset selection approaches clearly suggest that a hybrid model should be pursued [6]. In this this paper, we propose DoC which facilitates feature selection by ranking results and total frequency distribution, and applies it to the final selection process.

Feature ranking also called feature weighting, assesses individual features and assigns to them weights according to their degrees of relevance, while the feature selection (FS) evaluates the goodness of each found feature subset [6]. We considered FS methods as experts giving opinion on the ranking of the features. Each FS algorithm used has a different way of mathematical calculation thus has its own advantages and disadvantages.

sMRI is a widely available [7] and widely used neuroimaging technique in research as well as clinical practice [8]. Previous neuroimaging studies that used sMRI of depression patients reported certain patterns of brain changes that may be present at a structural levels [5]. Specifically, the image-based volumetric analysis of brain regions has drawn a lot of attention in depression related research in the past decade [5]. In depression detection, volumetric studies have identified reductions or increase in the hippocampus, amygdala, anterior cingulate cortex, orbitofrontal cortex, dorsolateral prefrontal cortex, subgenual prefrontal cortex, putamen, caudate and also cerebrospinal fluid. Therefore, there is a need to investigate the most relevant features for depression detection [5].

To date, there are several individual depression detection studies based on sMRI (Costafreda et al.[9], Nouretdinov et al. [10], Gong et al. [11], Mwangi et al. [12, 13], Bao et al. 